\section{Significance of Dynamic Imaging in Diagnosis of Hirayama Disease: A Rare Case Report and Literature Review}

Gautham R. Kandukuri, M.D., ${ }^{1,2}$ Natasha R. Acosta, M.D. ${ }^{1}$

${ }^{1}$ Truman Medical Center Hospital Hill, Department of Radiology, Kansas City, MO

${ }^{2}$ University of Missiouri-Kansas City, School of Medicine, Kansas City, MO

Received Oct. 3, 2019; Accepted for publication Dec. 12, 2019; Published online March 20, 2020

\section{INTRODUCTION}

In 1959, Hirayama et al. ${ }^{1}$ first described a benign, self-limiting cervical myelopathy, now known as Hirayama disease, monomelic amyotrophy, or juvenile spinal muscular atrophy of the distal upper extremity. ${ }^{2}$ Its usual presentation is unilateral progressive muscular weakness and wasting in the distal upper limb musculature with spontaneous arrest within several years. ${ }^{1}$ Often mistaken for a degenerative upper motor neuron disease, Hirayama disease is the result of asymmetric microcirculatory changes in the anterior horn cells of the distal cervical spinal cord resulting from repetitive neck flexion. ${ }^{2}$ Over time, the circulatory changes lead to cord atrophy, which manifests as upper extremity weakness. Although both flexor and extensor muscles of the hand are involved, the finger extensors and wrist flexors primarily are affected. The majority of patients develop unilateral amyotrophy, although some present with asymmetric or bilateral atrophy. ${ }^{3}$ Sensation and muscle stretch reflexes generally are preserved. The right upper limb is affected more frequently regardless of handedness. ${ }^{1}$ Muscle fatigue and contraction fasciculation are the most common presenting symptoms, followed by cold paresis, atrophy, and tremor., ${ }^{1,3}$ The disease predominantly presents in young males, in the teenage years or early twenties. ${ }^{3}$ There is no known genetic predisposition of the disease, and it rarely has familial occurrence. Most of the reported cases are from Japan and Asian countries, with little awareness of the disease in North America.

In this case report, an incidence of Hirayama disease that was diagnosed in North America is presented, its pathoetiology explored, and appropriate diagnostic techniques in everyday medical practice are discussed.

\section{CASE REPORT}

A 20-year-old male of Bhutanese descent, born in Nepal, presented to neurology clinic for evaluation of left-arm weakness, tremors, and atrophy. Onset of symptoms were three years prior, with gradual progression leading to functional impairment. Interestingly, the patient's older brother also suffered from progressive weakness in his left arm, which was significantly and functionally impaired. Of note, the patient denied any loss of sensation, numbness, tingling, bulbar symptoms, and weakness of other extremities, including his right arm.

On physical examination, the patient had notable loss of muscle bulk in his left forearm, both proximally and distally (forearm circumference: left proximal, $22.25 \mathrm{~cm}$, left distal, $15.5 \mathrm{~cm}$, right proximal, $24.10 \mathrm{~cm}$, right distal, $17.0 \mathrm{~cm}$ ). Mild tremor and dysmetria in fingernose-finger testing of the left hand was noted. Strength testing of upper extremities revealed diminished strength in biceps, triceps, brachioradialis, wrist extensors and flexors, finger extensors and flexors, and finger abductors of the left arm, when compared to the right arm. Sensation of touch, temperature, and vibration remained intact in bilateral upper extremities. There was no loss of tone, clonus, fasciculation or pronator drift noted in either arm. Deep tendon reflexes in all extremities and cranial nerve functions were intact bilaterally.

Electromyography (EMG) of the patient's extremities revealed reduced recruitments, as well as increased amplitude and duration of motor unit action potentials in all muscles of the left upper extremity, except the deltoid. The EMG findings were suggestive of a chronic disorder involving the motor neurons, axons, or both. Magnetic resonance images (MRI) of the brain and spinal cord (cervical, thoracic, and lumbar) were ordered. Imaging of the brain, thoracic, and lumbar spine were unremarkable. MRI of the cervical spine demonstrated asymmetric caliber of the cervical cord, which was smaller on the left. The asymmetry was best visualized on axial imaging and thought to relate to underlying cord atrophy. Mild disc bulges in the cervical spine (C3 through C7) with overall mild narrowing of the underlying thecal sac, but no significant spinal stenosis or mass effect upon the cord, were noted. Given the lack of radiological evidence for radiculopathy, the absence of generalized motor neuron disease, and the positive family history for asymmetric distal upper extremity weakness, there was high suspicion for monomyelic amyotrophy or Hirayama disease.

A confirmation MRI of the cervical spine in neck flexion was obtained. Flexion MRI showed diffuse narrowing of the thecal sac accentuated by neck flexion due to tightening of the dura along the dorsal aspect of the thecal sac. There was also re-demonstration of the asymmetric atrophy of the left half of the cervical spinal cord (Figure 1). Additionally, the enhancing dorsal venous plexus, from the level of C3 to the upper thoracic spinal canal, was noted to have prominent and diffuse engorgement on neck flexion, a finding consistent with Hirayama disease.

\section{DISCUSSION}

Although first described in 1959, ${ }^{1}$ due to its benign course, pathologic study of Hirayama disease was not available until 1982, when the first autopsy was conducted on a 38-year-old male who succumbed to lung cancer. ${ }^{4}$ Pathological study revealed an anterior-posterior flattening of the cervical cord, along with asymmetric left sided anterior horn atrophy and central necrosis, all of which were consistent with ischemic changes. ${ }^{1}$ Initial conventional neuroradiologic investigations, such as X-rays and CT myelograms, prior to 1970 s failed to reveal significant radiologic abnormalities due to their inability to provide dynamic imaging. However, advancement of imaging techniques in the 1980s, particularly magnetic resonance imaging, combined with knowledge of the pathophysiology of the disease, allowed investigators to use radiological imaging to observe dynamic changes in the spinal cord. Particularly, the MRI of cervical 
KANSAS JOURNAL of MEDICINE HIRAYAMA DISEASE

continued.

neck in flexion, confirming forward displacement of posterior dural wall of the lower cervical canal, became the standard diagnostic test in diagnosing Hirayama disease..$^{5-7}$

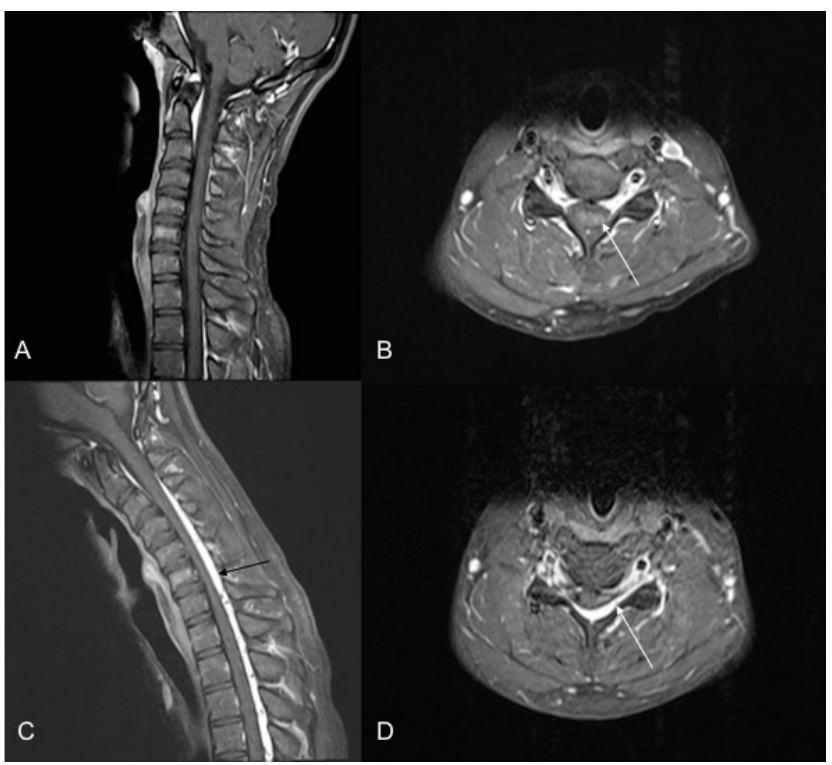

Figure 1. Patient's dynamic cervical spine MRI. Neutral position Tl post-contrast cervical spine MRI, sagittal image (A) and axial image at level of C6 (B) demonstrates mild asymmetric narrowing of the lower left cervical spinal cord (white arrow). Flexion Tl post-contrast cervical spine MRI, sagittal image (C) and axial image at level of C6 (D) demonstrates increased left-sided narrowing of the lower cervical spinal cord (white arrow) and exuberant enhancement of the dorsal venous plexus (black arrow), not observed on neutral views.

To understand the neuroradiological findings of Hirayama disease, it is important to familiarize oneself with its pathoetiology. While transitioning from neck extension to flexion, the length of the anterior dural wall, from the superior aspect of the atlas to the Tl vertebral body, increases approximately $1.5 \mathrm{~cm}$, whereas the posterior cervical dural wall stretches by almost $5 \mathrm{~cm}$ for the same degree of flexion. Normally, the posterior dura mater of the cervical spine is slack with transverse folds while the neck is in extension or neutral position. When the neck flexes, this laxity allows the dura to remain in close proximity to the vertebrae as the length of the vertebral canal increases. The posterior dural canal wall in patients with Hirayama disease is more prone to anterior displacement on neck flexion due to disproportionate lengths achieved by the vertebrae and dura mater during the adolescent growth phase in some individuals. ${ }^{8}$ The relatively shorter length ratio of the dura mater to vertebral column in Hirayama disease creates an "overstretching" of the posterior dural canal wall and causes anterior displacement of the dura on flexion. The displaced dura causes compression of the cord and leads to microcirculatory disturbances resulting in necrosis of the anterior horn cells over time with repeated flexion of the neck.

Cervical spine MRI in flexion and neutral positions, usually sagittal and axial views, is the most commonly used method of making the diagnosis of Hirayama disease. ${ }^{6}$ The dynamic flexion MRI has become the study of choice as it has a sensitivity of $87 \%$, which is increased greatly from the $49 \%$ sensitivity of the neutral position MRI. Apart from the obvious finding of anterior displacement of the posterior dura, there are several other flexion MRI findings characteristic of Hirayama disease. These include stretching or tightening of the dural sac, asymmetric flattening of the anterior lower cervical cord, subarachnoid space effacement, posterior epidural space enlargement forming a crescent shaped mass, epidural venous congestion manifesting as epidural flow voids, and enhancement of the epidural space on post-contrast studies. ${ }^{1}$

In our patient, flexion MRI showed distinct tightening of the dural sac and contrast enhancement of the venous plexus, both well documented Hirayama disease sequelae. ${ }^{1}$ The etiology for engorgement of the posterior venous plexus in cervical flexion is multifactorial and a prominent imaging finding in Hirayama disease. Cervical flexion causes anterior shift of the dural canal creating a negative pressure in the posterior spinal canal, compression of the anterior internal vertebral venous plexus diverting blood flow to the posterior venous plexus, and decrease in the drainage of the internal vertebral plexus into the jugular veins, all of which result in dilation of the posterior vertebral venous plexus. ${ }^{9}$

The non-flexion, neutral position MR imaging of cervical spine is the study most likely to be ordered by North American physicians evaluating unilateral cervical myelopathy, as they are less likely to be aware of Hirayama disease and the utility of the cervical flexion MRI in its diagnosis. ${ }^{6}$ Although generally considered less sensitive, there is evidence suggesting that neutral position MRI can be a very useful tool in diagnosis.

Chen et al. ${ }^{7}$ conducted a retrospective review of the MRIs of 46 patients with confirmed Hirayama disease to identify the sensitivity and specificity of neutral position image findings in disease diagnosis. They noted the following findings to be of particular usefulness in diagnosing Hirayama disease: abnormal cervical curvature, localized lower cervical cord atrophy, asymmetric cord flattening, loss of attachment between the posterior dural sac and subjacent lamina, and intramedullary high-signal intensity on T2-weighted MR images. According to their findings, loss of attachment between the posterior dural sac and the subjacent lamina has the highest combined sensitivity and specificity, at $93.5 \%$ and $98 \%$ respectively. Localized lower cervical cord atrophy, asymmetric cord flattening, and loss of attachment all had an accuracy of over $80 \%$ in detecting Hirayama disease.

Of interest, the possibility existed of a genetic or familial predisposition to Hirayama disease. Our patient had an older brother who had a significantly progressed right-handed weakness and atrophy, which had similar characteristics to Hirayama disease. Given that majority of the cases reported are in individuals of Asian or Japanese origin, there may be inherent susceptibility to the disease in certain genotypes. It also could be possible that the disease, having been discovered in Japan and researched extensively by investigators in Asia and Japan, is simply under diagnosed or misdiagnosed in North America due to lack of suspicion or knowledge.? 


\section{CONCLUSION}

The intent of this article was to familiarize North American clinicians and radiologists with Hirayama disease, its pathoetiology, and diagnostic neuroradiological techniques, using a case diagnosed in the region. Knowing the typical presentation and demographic prevalence of the disease should help interpret imaging studies better and suggest additional imaging techniques to arrive at the correct diagnosis. In the case of Hirayama disease, a dynamic MRI with neutral and flexion views can assist both the radiologist and clinician.

\section{REFERENCES}

${ }^{1}$ Huang YL, Chen CJ. Hirayama disease. Neuroimaging Clin N Am 2011; 2l(4):939-950. PMID: 22032508

2 Correia de Sá M, Costa H, Castro S, Vila Real M. A Portuguese case of Hirayama disease. BMJ Case Rep 2013; 2013. PMID: 24165502.

${ }^{3}$ Hirayama K. Juvenile muscular atrophy of distal upper extremity (Hirayama disease). Intern Med 2000; 39(4):283-290. PMID: 10801141.

${ }^{4}$ Gowda BN, Kumar MJ, Basim PK. Hirayama disease - A rare case report with review of literature. J Orthop Case Rep 2013; 3:11-14.

${ }^{5}$ Sitt J, Fung E, Yuen E, Ahuja A. Hirayama disease in a 17-year-old Chinese man. Singapore Medical Journal 2014; 55(6):10.

${ }^{6}$ Parihar A, Khurana N, Aga P, Singh R, Garg RK. Role of dynamic MRI study in Hirayama disease. Ann Indian Acad Neurol 2011; 14(2):138-139. PMID: 21808483.

${ }^{7}$ Chen CJ, Hsu HL, Tseng YC, et al. Hirayama flexion myelopathy: Neutralposition MR imaging findings - Importance of loss of attachment. Radiology 2004; 231(1):39-44. PMID: 15068939.

${ }^{8}$ Kikuchi S, Shinpo K, Niino M, Higashi T, Tashiro K. Cervical myelopathy due to a "tight dural canal in flexion" with a posterior epidural cavity. Intern Med 2002; 41(9):746-748. PMID: 12322806.

9 Vargas MC, Castlillo M. Magnetic resonance imaging in Hirayama disease.

J Radiol Case Rep 2011; 5(3):17-23. PMID: 22470782.

Keywords: Hirayama disease, monomelic amyotrophy, spinal muscular atrophy, distal, with upper limb predominance
KANSAS JOURNAL of MEDICINE

HIRAYAMA DISEASE

continued. 